LA GRANJA:

REVISTA DE

CIENCIAS DE LA VIDA

pISSN:1390-3799; eISSN:1390-8596

http:/ / doi.org/10.17163/lgr.n33.2021.09
Artículo científico / Scientific paper

ACUACUltura SUSTENTABLE

\title{
EVALUACIÓN DEL RIESGO AMBIENTAL POR LOS INSECTICIDAS FIPRONIL E IMIDACLOPRID EN EL CAMARÓN DE RÍO (Cryphiops caementarius)
}

\author{
ENVIRONMENTAL RISK ASSESSMENT BY FIPRONIL AND IMIDACLOPRID \\ INSECTICIDES IN RIVER SHRIMP (Cryphiops caementarius)
}

Fiorella Alexandra Cáceres-Del Carpio ${ }^{1}\left[\begin{array}{l}{[0} \\ \text { y José Iannacone* }\end{array}\right.$

\begin{abstract}
${ }^{1}$ Laboratorio de Ingeniería Ambiental, Carrera de Ingeniería Ambiental. COEPERU - Coastal Ecosystems of Peru Research Group. Universidad Científica del Sur, Carr. Panamericana Sur 19, Villa EL Salvador, 15067, Perú.

${ }^{2}$ Grupo de Investigación Sostenibilidad Ambiental (GISA), Laboratorio de Ecología y Biodiversidad Animal (LEBA). Escuela Universitaria de Posgrado, Universidad Nacional Federico Villarreal, El Agustino, 15007, Lima, Perú.
\end{abstract}

*Autor para correspondencia: jiannacone@cientifica.edu.pe

Manuscrito recibido el 9 de mayo de 2020. Aceptado, tras revisión, el 11 de junio de 2020. Publicado el 1 de marzo de 2021.

\begin{abstract}
Resumen
En la actualidad, el fipronil y el imidacloprid representan aproximadamente un tercio del mercado mundial de insecticidas. En el presente estudio se evaluó el riesgo ambiental (ERA) del fipronil e imidacloprid en las postlarvas del camarón de río (Cryphiops caementarius, Molina 1782). Se realizaron bioensayos de toxicidad de corta duración en base a la $\mathrm{CL}_{50}$ (Concentración letal media) (mortalidad) y $\mathrm{CE}_{50}$ (Concentración efectiva media) (hipoactividad natatoria). Se calculó la PNEC (Concentración prevista sin efecto conocido) y los estándares ambientales disponibles para la PEC (Concentración ambiental esperada) para el fipronil y el imidacloprid para determinar los cocientes de riesgo (CR). El imidacloprid resultó con mayor riesgo para el ambiente acuático que el fipronil para la respuesta letal (mortalidad) y subletal (hipoactividad natatoria). La diferencia del riesgo observada entre ambos insecticidas pudiera deberse a sus diferentes modos de acción. C. caementarius debería ser considerado como una especie sensible al momento de definir un estándar de calidad ambiental para la conservación del ambiente acuático. Por ende, es recomendable continuar el monitoreo para observar la presencia de estos insecticidas en los ecosistemas dulceacuícolas costeros, y reducir el uso del fipronil y del imidacloprid en los cultivos agrícolas que los emplean.
\end{abstract}

Palabras clave: Calidad ambiental, Cryphiops caementarius, Ecosistema acuático, Fipronil, Hipoactividad natatoria, Imidacloprid. 


\begin{abstract}
Fipronil and imidacloprid currently represent approximately one third of the global insecticide market. In the present study, the environmental risk (ERA) of fipronil and imidacloprid in the postlarvae of the river shrimp (Cryphiops caementarius, Molina 1782) was evaluated. Short-term toxicity bioassays were performed based on $\mathrm{LC}_{50}$ (mean lethal concentration) (mortality) and $\mathrm{EC}_{50}$ (mean effective concentration) (swimming hypoactivity). PNEC (Predicted Concentration with No Known Effect) and available environmental standards for PEC (Expected Environmental Concentration) were calculated for fipronil and imidacloprid to determine risk quotient (RQ). Imidacloprid was more at risk for the aquatic environment than fipronil for the lethal response (mortality) and sublethal response (swimming hypoactivity). The observed risk difference between the two insecticides could be due to their different modes of action. C. caementarius should be considered as a sensitive species when defining an environmental quality standard for the conservation of the aquatic environment. Therefore, it is recommended to continue monitoring the presence of these insecticides in coastal freshwater bodies, and to reduce the use of fipronil and imidacloprid in the agricultural crops that use them.
\end{abstract}

Keywords: Environmental quality, Cryphiops caementarius, Aquatic ecosystem, Fipronil, swimming hypoactivity, Imidacloprid

Forma sugerida de citar: Cáceres-Del Carpio, F.A. y Iannacone, J. (2021). Evaluación del Riesgo Ambiental por los insecticidas Fipronil e Imidacloprid en el Camarón de río (Cryphiops caementarius). La Granja: Revista de Ciencias de la Vida. Vol. 33(1):104-114. http:/ /doi.org/10.17163/ lgr.n33.2021.09.

IDs Orcid:

Fiorella Alexandra Cáceres-Del Carpio: http:/ / orcid.org/0000-0001-5149-6681

José Iannacone: http:/ / orcid.org/0000-0003-3699-4732

La Granja: Revista de Ciencias de la Vida 33(1) 2021:104-114.

(C2021, Universidad Politécnica Salesiana, Ecuador. 


\section{Introducción}

La importación de insecticidas en el Perú aumentó de 3481 tn a 5037 tn entre los años 2007 a 2017 (INEI, 2018), y debido a sus impactos ecológicos en los cuerpos de agua y la afectación a la biodiversidad acuática (Escobar-Chávez, Alvariño y Iannacone, 2019; Sotelo-Vásquez y Iannacone, 2019), es que el uso de los plaguicidas se regula para la protección de la salud y el medio ambiente por el Servicio Nacional de Sanidad Agraria (MINAGRI, 2020). Actualmente, el fipronil e imidacloprid representan aproximadamente un tercio del mercado mundial de insecticidas (MINAGRI, 2020). El fipronil y el imidacloprid actúan bloqueando la transmisión en el sistema nervioso central, pero cada sustancia química tiene un modo de acción diferente (Al-Badran y col., 2018; Al-Badran, Fujiwara y Mora, 2019). El fipronil interfiere con el paso de iones cloruro al unirse a un sitio específico dentro del receptor de ácido gamma-aminobutírico (GABA), mientras que el imidacloprid se une a los receptores de acetilcolina nicotínicos postsinápticos (nAChR) (Al-Badran y col., 2018).

El fipronil (fenilpirazoles) e imidacloprid (neonicotinoides) se aplican sobre plagas agrícolas, plagas domésticas y ectoparásitos de los animales domésticos (Al-Badran y col., 2018; Al-Badran, Fujiwara y Mora, 2019; Escobar-Chávez, Alvariño y Iannacone, 2019). En el Perú, en los cultivos agrícolas de arroz y cebolla, los insecticidas fipronil e imidacloprid son ampliamente empleados para el control de plagas (Gangwar y col., 2016; Pathak y col., 2018). En comparación con otros tipos de insecticidas, el fipronil y el imidacloprid se consideran más seguros debido a su baja toxicidad en peces y mamíferos. El fipronil y el imidacloprid son muy efectivos sobre los artrópodos en pequeñas concentraciones (AlBadran y col., 2018). El incremento de su empleo en décadas recientes, la solubilidad moderada a alta y la persistencia en el agua plantean una seria preocupación con relación a los posibles efectos negativos en los invertebrados acuáticos que no son el objetivo del control (Al-Badran, Fujiwara y Mora, 2019).

El camarón de río (Cryphiops caementarius, Molina 1782), es una especie de relevancia biológica, comercial y económica en el Perú, y constituye uno de los componentes hidrobiológicos endémicos que mantiene la pesquería comercial de la costa sur del
Perú y norte de Chile (Campos y col., 2017). Se han registrado sus más altas poblaciones en los ríos del departamento de Arequipa (Perú), debido a que estos cuerpos de agua presentan un mayor caudal, además del predominio en esta zona sobre el cultivo de arroz y cebolla. Cryphiops caementarius se emplea en la acuicultura para la alimentación (Reyes, 2011; Reyes, 2018; Romero-Camarena, Zelada y Álvarez, 2013; Campos y col., 2017), sometido a una explotación hidrobiológica alta en el medio natural, al presentarse impactos sobre la destrucción de su hábitat por procesos naturales de sequía, y antrópicos como el empleo del agua, actividades agrícolas y mineras (Wasiw, 2017).

A la fecha no existe ninguna investigación que evalúe el riesgo ambiental de insecticidas al emplear a C. caementarius como especie bioindicadora (Smit y col., 2015; Al-Badran y col., 2018; Al-Badran, Fujiwara y Mora, 2019). Por lo tanto, el objetivo del presente trabajo fue evaluar el riesgo ambiental de los insecticidas fipronil e imidacloprid en C. caementarius.

\section{Materiales y Métodos}

\subsection{Camarón de río Cryphiops caementa- rius (Molina 1782)}

Las postlarvas del camarón de río (C. caementarius) fueron obtenidas del río Cañete en el distrito de Calango, Cañete, Lima, Perú $\left(12^{\circ} 31^{\prime} 35^{\prime \prime} \mathrm{S} 76^{\circ} 32^{\prime} 38^{\prime \prime} \mathrm{O}\right)$ por un pescador autorizado perteneciente al gremio de pescadores de la zona (Resolución N ${ }^{\circ} 83-2007-$ PRODUCE), Perú. Las postlarvas se obtuvieron en el periodo de ausencia de veda de esta especie (Baltazar y Colón, 2014; Wasiw y Yépez, 2015).

Se aclimataron las postlarvas de C. caementarius en el Laboratorio de Larvicultura de la Universidad Científica del Sur (UCSUR), Lima, Perú, dos semanas antes del inicio de los bioensayos y siguiendo el siguiente esquema: cerca de 1000 postlarvas se mantuvieron en un contenedor de $750 \mathrm{~L}$ de capacidad con aireación constante a una temperatura promedio de $21 \pm 2{ }^{\circ} \mathrm{C}$, suministrando hígado de pollo precocido como alimento cada $24 \mathrm{~h}$ para evitar la muerte de los organismos. Se realizó diariamente el cambio de agua, la cual fue previamente declorada empleando tiosulfado de sodio mediante sifoneo (Rice, Baird y Eaton, 2017). Para los bioensayos 
se utilizaron postlarvas de $C$. caementarius con talla promedio de $15 \mathrm{~mm}$ (Baltazar y Colón, 2014). Se usó un calibrador $( \pm 0,1 \mathrm{~mm})$ para medir la longitud total de cada postlarva de C. caementarius al enderezar el cuerpo del camarón cuidadosamente sobre la mesa y midiendo la longitud total desde la punta de la cabeza hasta el final de la cola (Al-Badran, Fujiwara y Mora, 2019).

\subsection{Insecticidas}

Se empleó el fipronil de la marca Regent SC a una concentración de $250 \mathrm{~g} \cdot \mathrm{L}^{-1}$ y se establecieron siete concentraciones nominales $\left(0,10 \mu \mathrm{g} \cdot \mathrm{L}^{-1}\right.$; $0,26 \mu \mathrm{g} \cdot \mathrm{L}^{-1} ; 0,64 \mu \mathrm{g} \cdot \mathrm{L}^{-1} ; 1,6 \mu \mathrm{g} \cdot \mathrm{L}^{-1} ; 4 \mu \mathrm{g} \cdot \mathrm{L}^{-1} ; 0,02$ $\mu \mathrm{g} \cdot \mathrm{L}^{-1}$ y $\left.0,04 \mu \mathrm{g} \cdot \mathrm{L}^{-1}\right)$. Para el imidacloprid se utilizó la marca Imidamin a una concentración de 350 $\mathrm{g} \cdot \mathrm{L}^{-1}$ con cinco concentraciones nominales $(28,8$ $\mu \mathrm{g} \cdot \mathrm{L}^{-1} ; 71,9 \mu \mathrm{g} \cdot \mathrm{L}^{-1} ; 179,8 \mu \mathrm{g} \cdot \mathrm{L}^{-1} ; 449,6 \mu \mathrm{g} \cdot \mathrm{L}^{-1}$ y $\left.1124 \mu \mathrm{g} \cdot \mathrm{L}^{-1}\right)$.

\subsection{Bioensayos}

Se usaron cuatro repeticiones por cada insecticida, más un control. Se colocaron $2 \mathrm{~L}$ con agua de dilución a contenedores con una capacidad de $3 \mathrm{~L}$, los cuales se conectaron a un sistema con aireación constante en serie, para cada concentración y repetición. Luego se colocaron 10 ejemplares de postlarva del C. caementarius en cada uno de los contenedores (Escobar-Chávez, Alvariño y Iannacone, 2019). El agua que se usó en los contenedores se acondicionó con Nutrafin Aqua Plus (Hagen, USA).

Las postlarvas de camarón de río se alimentaron cada $48 \mathrm{~h}$ con arroz cocido para evitar su muerte por canibalismo. Las mediciones de mortalidad e hipoactividad natatoria postlarval (hNPL) se realizaron a las 3h, 8h, 24h, 48h, 72h y 96h de exposición. Los organismos fueron considerados muertos ante la ausencia total de movimiento durante 2 min luego de tocarse suavemente con la ayuda de un paliglobo. La hNPL se catalogó como la falta de desplazamiento, falta de lucha, ausencia de reacción a estímulos mecánicos, y aletargamiento. La actividad natatoria postlarval normal fue considerada como la búsqueda de alimento, desplazamiento en toda la columna de agua y reacción rápida a estímulos mecánicos.

\subsection{Análisis de datos y evaluación del ries- go ambiental}

Se determinaron los porcentajes de mortalidad e hNPL de C. caementarius. La concentración letal media $\left(\mathrm{CL}_{50}\right)$ para la mortalidad y la concentración efectiva media $\left(\mathrm{CE}_{50}\right)$ para la hNPL fueron calculadas con el programa Probit versión 1,5 con un nivel de confianza al $95 \%$ y el modelo de regresión se verificó con el estadístico Chi-cuadrado $\left(\chi^{2}\right)$ (Rice, Baird y Eaton, 2017). Los parámetros LOEC (concentración más baja donde se observa efecto) y NOEC (concentración de efecto no observado) se calcularon con el programa estadístico Past 3.2, mediante la prueba de Krustal-Wallis en base a las diferencias significativas entre las mortalidades y las hNPLs para las concentraciones del fipronil e imidacloprid empleadas.

\subsubsection{Concentración prevista sin efecto conocido (PNEC)}

Se halló la PNEC a partir de la CL(E) $)_{50}$, LOEC y NOEC derivados de los resultados de los ensayos de toxicidad de corta duración. A estos parámetros se le aplicó el "factor de valoración" (FV) o seguridad establecidos para ensayos de toxicidad, el cual fue de 1000 (PNUMA/IPCS1999). Con PNEC la relación entre parámetros de toxicidad/Factor de valoración.

\subsubsection{Concentración ambiental esperada (PEC)}

Se emplearon los estándares de calidad ambiental (ECA) de la legislación de los Países Bajos para el fipronil (Tennekes, 2018) y para el imidacloprid (Smit y col., 2015), presentando los ECA del imidacloprid dos escenarios. Escenario 1: ECA a corto plazo, de concentración máxima aceptable (MAC-ECA) basado en datos de toxicidad aguda. Escenario 2: ECA a largo plazo, expresado como una concentración promedio anual (AA-ECA) basado en datos de toxicidad crónica con el objetivo de proteger el ecosistema contra los efectos adversos resultantes de la exposición a largo plazo (EC, 2011).

\subsubsection{Caracterización del riesgo (PEC/PNEC)}

Se calculó el cociente de riesgo $(\mathrm{CR})$, que establece que si la PEC/PNEC es $<1$ se considera que el fipronil e imidacloprid evaluados son de bajo riesgo; mientras que cuando PEC/PNEC $>1$, se considera 
un alto riesgo (De la Torre, Ñuñoz y Carballo, 2004). Con CR la relación entre PEC/PNEC.

\section{Resultados}

La mortalidad y la hNPL aumentan desde las $3 \mathrm{~h}$ hasta las $96 \mathrm{~h}$ de exposición en las postlarvas de C. caementarius que presentaron a las $96 \mathrm{~h}$ de exposición un mayor efecto en el fipronil, llegando al $100 \%$ de mortalidad y de hNPL. Para el imidaclo- prid se alcanzó un $87,5 \%$ y $100 \%$ para la mortalidad y el hNPL, respectivamente. Se obtuvieron los valores de la $\mathrm{CL}_{50}$ y $\mathrm{CE}_{50}$ para los insecticidas fipronil con sus límites superiores e inferiores al 95\% desde las $3 \mathrm{~h}$ hasta las $96 \mathrm{~h}$ de exposición, y sus respectivos coeficientes de determinación ( $\mathrm{R}^{2}$ ) (Tablas 1 y 2). De igual forma se observan los parámetros de LOEC y NOEC para el fipronil e imidacloprid desde las $3 \mathrm{~h}$ hasta las $96 \mathrm{~h}$ de exposición en las postlarvas de $C$. caementarius (Tabla 3 y 4).

Tabla 1. Concentración letal media $\left(\mathrm{CL}_{50}\right)$ y Concentración efectiva media $\left(\mathrm{CE}_{50}\right)$ y límites superiores e inferiores para parámetros letales y subletales en base a la hNPL (hipoactividad natatoria) en Cryphiops caementarius para el fipronil a seis diferentes tiempos de exposición.

\begin{tabular}{ccccc}
\hline $\begin{array}{c}\text { Tiempo de exposición } \\
(\mathbf{h})\end{array}$ & $\begin{array}{c}\text { Mortalidad CL } \\
\left(\mu \mathbf{g} \cdot \mathbf{L}^{-1}\right)\end{array}$ & $\mathbf{R}^{2}$ & $\begin{array}{c}\text { hNPL CE } \\
\left(\mu \mathbf{g} \cdot \mathbf{L}^{-1}\right)\end{array}$ & $\mathbf{R}^{2}$ \\
\hline $3 \mathrm{~h}$ & $0,901(0,506-1,601)$ & 0,98 & $0,252(0,175-0,364)$ & 0,99 \\
$8 \mathrm{~h}$ & $0,679(0,354-1,303)$ & 0,97 & $0,074(0,042-0,131)$ & 0,96 \\
$24 \mathrm{~h}$ & $0,035(0,007-0,171)$ & 0,87 & $0,003(0,001-0,007)$ & 0,99 \\
$48 \mathrm{~h}$ & $<0,02$ & $\mathrm{ND}$ & $<0,02$ & $\mathrm{ND}$ \\
$72 \mathrm{~h}$ & $<0,02$ & $\mathrm{ND}$ & $<0,02$ & $\mathrm{ND}$ \\
$96 \mathrm{~h}$ & $<0,02$ & $\mathrm{ND}$ & $<0,02$ & $\mathrm{ND}$ \\
\hline
\end{tabular}

( ): Límites superiores e inferiores $(95 \%)$. $\mathrm{R}^{2}$ : coeficiente de determinación.

Tabla 2. Concentración letal media $\left(\mathrm{CL}_{50}\right)$ y concentración efectiva media $\left(\mathrm{CE}_{50}\right)$ y límites superiores e inferiores para parámetros letales (mortalidad) y subletales en base a la hNPL (hipoactividad natatoria) en Cryphiops caementarius para el imidacloprid a seis diferentes tiempos de exposición.

\begin{tabular}{ccccc}
\hline $\begin{array}{c}\text { Tiempo de exposición } \\
(\mathbf{h})\end{array}$ & $\begin{array}{c}\text { Mortalidad CL } \\
\left(\mu \mathbf{g} \cdot \mathbf{L}^{-1}\right)\end{array}$ & $\mathbf{R}^{2}$ & $\begin{array}{c}\mathbf{h N P L} \mathbf{C E}_{50} \\
\left(\mu \mathbf{g} \cdot \mathbf{L}^{-1}\right)\end{array}$ & $\mathbf{R}^{2}$ \\
\hline $3 \mathrm{~h}$ & $\mathrm{ND}$ & $\mathrm{ND}$ & $260,5(82,97-817,92)$ & 0,83 \\
$8 \mathrm{~h}$ & $\mathrm{ND}$ & $\mathrm{ND}$ & $246,7(152,42-399,42)$ & 0,94 \\
& 5353,7 & 1 & $28,4(6,62-122,17)$ & 0,97 \\
$48 \mathrm{~h}$ & $(1832,17-15643,56)$ & & $5,3(1,11-25,66)$ & 0,46 \\
$72 \mathrm{~h}$ & $53540,8(1032,89-2775350,23)$ & 0,52 & $1,2(0,14-9,83)$ & 0,93 \\
$96 \mathrm{~h}$ & $13,68(1,72-109,11)$ & 0,97 & $0,002(0,00-0,16)$ & 0,79 \\
\hline
\end{tabular}

( ): Límites superiores e inferiores al $95 \% . \mathrm{R}^{2}$ : coeficiente de determinación.

La Tabla 5 muestra los valores que establecen la relación entre la PEC y el PNEC para determinar el riesgo existente de los insecticidas, basados en los

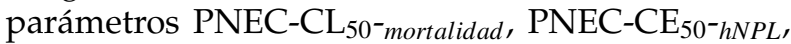
PNEC-LOEC-mortalidad y PNEC-LOEC- ${ }_{h N P L}$. Para el fipronil y para el imidacloprid se obtuvieron valo- res de CRs mayores a uno en todos los casos (Tabla 5). El imidacloprid presentó un mayor riesgo para el ambiente acuático que el fipronil para la respuesta letal (mortalidad) y subletal (hipoactividad natatoria postlarval). 
Tabla 3. Concentración más baja donde se observa efecto (LOEC) y concentración de efecto no observado (NOEC) para parámetros letales (mortalidad) y subletales en base a la hNPL (hipoactividad natatoria) en Cryphiops caementarius para el fipronil a seis diferentes tiempos de exposición.

\begin{tabular}{ccccc}
\hline $\begin{array}{c}\text { Tiempo de } \\
\text { exposición } \\
(\mathbf{h})\end{array}$ & \multicolumn{2}{c}{$\begin{array}{c}\text { Mortalidad } \\
\left(\mu \mathbf{g} \cdot \mathbf{L}^{-1}\right)\end{array}$} & \multicolumn{2}{c}{$\mathbf{h N P L}$} \\
$\left.\mathbf{L O E} \cdot \mathbf{L}^{-1}\right)$ & & NOEC & LOEC & NOEC \\
\hline $3 \mathrm{~h}$ & 1,60 & 0,64 & 0,26 & 0,10 \\
$8 \mathrm{~h}$ & 0,26 & 0,10 & 0,04 & 0,02 \\
$24 \mathrm{~h}$ & 0,02 & $<0,02$ & 0,02 & $<0,02$ \\
$48 \mathrm{~h}$ & 0,02 & $<0,02$ & 0,02 & $<0,02$ \\
$72 \mathrm{~h}$ & 0,02 & $<0,02$ & 0,02 & $<0,02$ \\
$96 \mathrm{~h}$ & 0,02 & $<0,02$ & 0,02 & $<0,02$ \\
\hline \multicolumn{4}{l}{ LOEC: Concentración más baja donde se observa efecto. } \\
NOEC: Concentración de efecto no observado.
\end{tabular}

Tabla 4. Concentración más baja donde se observa efecto (LOEC) y concentración de efecto no observado (NOEC) para parámetros letales (mortalidad) y subletales en base a la hNPL (hipoactividad natatoria) en Cryphiops caementarius para el imidacloprid a diferentes tiempos de exposición.

\begin{tabular}{ccccc}
\hline $\begin{array}{c}\text { Tiempo de } \\
\text { exposición } \\
(\mathbf{h})\end{array}$ & \multicolumn{2}{c}{$\begin{array}{c}\text { Mortalidad } \\
\left(\mu \mathbf{g} \cdot \mathbf{L}^{-1}\right)\end{array}$} & \multicolumn{2}{c}{$\mathbf{h N P L}$} \\
$\mathbf{\text { LOEC }}$ & NOEC & $\left.\mathbf{L} \mathbf{L}^{-1}\right)$ \\
\hline $3 \mathrm{~h}$ & 28,8 & $<28,8$ & 179,8 & NOEC \\
\hline $8 \mathrm{~h}$ & 28,8 & $<28,8$ & 179,8 & 71,9 \\
$24 \mathrm{~h}$ & 28,8 & $<28,8$ & 28,8 & $<28,8$ \\
$48 \mathrm{~h}$ & 28,8 & $<28,8$ & 28,8 & $<28,8$ \\
$72 \mathrm{~h}$ & 28,8 & $<28,8$ & 28,8 & $<28,8$ \\
$96 \mathrm{~h}$ & 28,8 & $<28,8$ & 28,8 & $<28,8$ \\
\hline
\end{tabular}

LOEC: Concentración más baja donde se observa efecto. NOEC: Concentración de efecto no observado.

\section{Discusión}

No se han realizado bioensayos de toxicidad con el fipronil y el imidacloprid en C. caementarius, pero se observan investigaciones con otras especies de crustáceos acuáticos (Goff y col., 2017; Al-Badran, Fujiwara y Mora, 2019). C. caementarius presentó efectos en la mortalidad y en la hipoactividad natatoria (hNPL) para ambos insecticidas, en la hNPL se manifestó falta de reacción ante estímulos brindados, reducción del movimiento y nado extraño, los resultados obtenidos variaron con las concentraciones y tiempos de exposición en ambos insecticidas (Mendoza-Rodríguez, 2009).

Shan y col. (2003) encontraron para el fipronil en Macrobrachium rosenbergii (De Man, 1879) y Macrobrachium niponnensis (De Haan, 1849), valores de $\mathrm{CL}_{50}(24 \mathrm{~h}) \mathrm{de} 6,41 \mu \mathrm{g} \cdot \mathrm{L}^{-1} \mathrm{y}>25,70 \mu \mathrm{g} \cdot \mathrm{L}^{-1}$, respecti- vamente. C. caementarius fue más sensible al fipronil que las dos especies de Macrobrachium con un valor de $\mathrm{CL}_{50}$ de $0,035 \mu \mathrm{g} \cdot \mathrm{L}^{-1}$ a las $24 \mathrm{~h}$ de exposición. Las diferencias en las $\mathrm{CL}_{50}$ obtenidas, pueden deberse a los diferentes protocolos empleados en los bioensayos, donde Shan y col. (2003) realizaron una simulación de los campos de arroz en condiciones de laboratorio. También estas diferencias pueden ser explicadas por los patrones específicos de biodistribución, a las tasas de metabolización, o incluso a la sensibilidad específica de cada taxón objetivo.

El fipronil e imidacloprid en Palaemonetes pugio Holthuis, 1949 a las 96 h de exposición obtuvo una $\mathrm{CL}_{50}$ de $0,68 \mu \mathrm{g} \cdot \mathrm{L}^{-1}$ para las larvas y una $\mathrm{CL}_{50}$ de $0,32 \mu \mathrm{g} \cdot \mathrm{L}^{-1}$ para los adultos ante el fipronil, y para el imidacloprid fue significativamente más tóxica en las larvas $\left(\mathrm{CL}_{50}\right.$ de $\left.308 \mu \mathrm{g} \cdot \mathrm{L}^{-1}\right)$ que en los adultos (CL50 de 563,5 $\mu \mathrm{g} \cdot \mathrm{L}^{-1}$ ) (Key y col., 2007). 
Tabla 5. Valores de PEC (Evaluación de la Exposición), PNEC (concentración prevista sin efecto conocido) para determinar el CR (Cociente de riesgo) de los insecticidas fipronil e imidacloprid empleando al camarón de rio Cryphiops caementarius.

\begin{tabular}{|c|c|c|c|}
\hline Parámetros & Fipronil & $\begin{array}{l}\text { Imidacloprid- } \\
\text { Escenario } 1\end{array}$ & $\begin{array}{l}\text { Imidacloprid- } \\
\text { Escenario } 2\end{array}$ \\
\hline PEC & 0,00007 (ECA) & 0,2 (MAC-ECA ) & 0,0083 (AA-ECA) \\
\hline PNEC ( $\left.\mathrm{CL}_{50^{-} \text {mortalidad }}\right)$ & 0,00002 & 0,00023 & 0,00023 \\
\hline PNEC $\left(\mathrm{CE}_{50}{ }^{-} h N P L\right)$ & 0,00002 & 0,000002 & 0,000002 \\
\hline PNEC (LOEC- $\left.{ }_{\text {mortalidad }}\right)$ & 0,00002 & 0,028 & 0,028 \\
\hline PNEC (LOEC- $h N P L)$ & 0,00002 & 0,028 & 0,028 \\
\hline $\mathrm{CR}\left(\mathrm{CL}_{50-\text { mortalidad }}\right)$ & 3,5 & 869,56 & 36,08 \\
\hline $\mathrm{CR}\left(\mathrm{CE}_{50-h N P L}\right)$ & 3,5 & 100.000 & 4.150 \\
\hline CR (LOEC-mortalidad $)$ & 3,5 & 7,14 & 2,96 \\
\hline $\mathrm{CR}(\mathrm{LOEC}-h N P L)$ & 3,5 & 7,14 & 2,96 \\
\hline
\end{tabular}

$\mathrm{ECA}=$ Estándar de Calidad Ambiental. $\mathrm{CL}_{50}=$ Concentración letal media a 96 h de exposición. $\mathrm{CE}_{50}=$ Concentración Efectiva media a 96 h de exposición. $\mathrm{LOEC}=$ concentración más baja donde se observa efecto a 96 h de exposición. MAC-ECA= Concentración máxima permisible para un Estándar de Calidad Ambiental a corto plazo. AA-ECA= ECA a largo plazo, expresado como una concentración promedio anual.

En el caso del C. caementarius, la exposición al fipronil y al imidacloprid a las 96 h de exposición para la mortalidad en postlarvas de camarón evidenció diferencia con la investigación de Key y col. (2007). Se hallaron también los parámetros de LOEC y NOEC para la mortalidad, los cuales fueron de $0,02 \mu \mathrm{g} \cdot \mathrm{L}^{-1}$ y $<0,02 \mu \mathrm{g} \cdot \mathrm{L}^{-1}$ a las $96 \mathrm{~h}$ de exposición para el fipronil, y de $28,8 \mu \mathrm{g} \cdot \mathrm{L}^{-1} \mathrm{y}<28,8 \mu \mathrm{g} \cdot \mathrm{L}^{-1}$ (96 h) para el imidacloprid, respectivamente. Key y col. (2007), encontraron valores de menor toxicidad para $P$. pugio que los de la presente investigación.

El fipronil presentó mayor toxicidad letal que el imidacloprid en base a la $\mathrm{CL}_{50}$ C. caementarius a 96 h de exposición. Omar y col. (2016), en Marsupenaeus japonicus (Spence Bate, 1888), encontraron efectos variables de acuerdo al estadio de desarrollo ensayado. La mayor toxicidad letal del fipronil en comparación al imidacloprid se ha observado en otras especies de crustáceos decápodos como Farfantepenaeus aztecus (Al-Badran, Fujiwara y Mora, 2019). Penaeus monodon Fabricius, 1798 fue sometido al fipronil y al imidacloprid en el estadio de postlarvas a las $48 \mathrm{~h}$ de exposición, encontrando valores de $\mathrm{CL}_{50}$ de $0,2 \mu \mathrm{g} \cdot \mathrm{L}^{-1}$ y $175 \mu \mathrm{g} \cdot \mathrm{L}^{-1}$, respectivamente (Hook y col., 2018).

Los artrópodos se encuentran entre los taxones más sensibles al fipronil, y las especies relacionadas pueden tener sensibilidades muy variadas a este in- secticida (Stevens y col., 2011), y esto se debe a que el fipronil puede ser más tóxico una vez metabolizado, debido a que el sulfuro de fipronilo y la sulfona de fipronilo son generalmente dos a tres veces más tóxicos que el compuesto original. Asimismo, existe una gama muy amplia en la sensibilidad de los crustáceos al imidacloprid, con valores de $\mathrm{CL}_{50}$ que varían de 1 a $52500 \mu \mathrm{g} \cdot \mathrm{L}^{-1}$ (Smit y col., 2015).

Se han observado efectos subletales por acción del fipronil e imidacloprid en el comportamiento, fisiología, reproducción y desarrollo de invertebrados acuáticos no destinatarios (Al-Badran y col., 2018; Sohn y col., 2018). Para el parámetro subletal de hNPL, se encontró una $\mathrm{CL}_{50}$ en un rango de $260,5 \mu \mathrm{g} \cdot \mathrm{L}^{-1}$ a $0,002 \mu \mathrm{g} \cdot \mathrm{L}^{-1}$, entre las $3 \mathrm{~h}$ y 96 $\mathrm{h}$ para el imidacloprid y un rango de $0,252 \mu \mathrm{g} \cdot \mathrm{L}^{-1}$ y $<0,02 \mu \mathrm{g} \cdot \mathrm{L}^{-1}$ obtenidos entre las $3 \mathrm{~h}$ y $96 \mathrm{~h}$ de exposición para el fipronil. El fipronil, a diferencia del imidacloprid, causó nado errático en todas las direcciones o convulsiones y reacción inmediata ante el estímulo del movimiento, mientras que ante el imidacloprid las postlarvas presentaron aletargamiento, disminución del nado en la columna de agua, y reacción tardía ante la estimulación del movimiento.

Para ambos insecticidas se presentó lucha por el alimento, ausencia total del desplazamiento antes de la muerte, pese al intento de nado que se reflejó en la movilidad de los apéndices locomotores. Al- 
Badran, Fujiwara y Mora (2019) encontraron cambios en el comportamiento de F. aztecus por acción del fipronil e imidacloprid bajo diferentes tiempos de exposición en dependencia de las concentraciones. El imidacloprid redujo el comportamiento de defensa del crustáceo Orconecres rusticus (Sohn y col., 2018). En el presente trabajo el imidacloprid presentó mayores efectos subletales que el fipronil.

Los diferentes efectos que presentaron las postlarvas ante ambos insecticidas se deben a los modos de acción de cada uno. El fipronil es un antagonista del GABA que provoca hiperexcitación y convulsiones; mientras que el imidacloprid es un antagonista del nAChR que causa una variedad de síntomas desde hiperexcitación hasta letargo y parálisis (Cox y col., 1998; Al-Badran, Fujiwara y Mora, 2019).

Para los ingredientes activos del fipronil e imidacloprid, los resultados obtenidos con el CR de corta duración muestran la existencia de un riesgo ambiental y concuerda con los estudios de Sluijs y col. (2015), que muestran los riesgos para la biodiversidad y el funcionamiento del ecosistema por el uso generalizado de neonicotinoides como el imidacloprid y fipronil. Se han encontrado que muestras tomadas en aguas subterráneas y superficiales superan los límites basados en los umbrales ecológicos establecidos en diferentes países de América del Norte y Europa, lo cual indica que existen en los suelos, las vías fluviales y las plantas en entornos agrícolas, urbanos y en áreas de drenaje que están contaminadas con mezclas de fipronil, neonicotinoides o sus metabolitos (Bonmatin y col., 2015). Sluijs y col. (2015) muestra una creciente evidencia que estos insecticidas representan un alto riesgo para una amplia gama de taxones de invertebrados no objetivos, lo cual tendría un impacto en las cadenas alimenticias acuáticas.

Los plaguicidas pueden ser lixiviados en zanjas y ríos por las lluvias, y las aguas superficiales se pueden contaminar con un rociado directo, mediante la escorrentía y lixiviación de los campos agrícolas (Vijver y Brink, 2014). La emisión a las aguas superficiales del fipronil e imidacloprid están originadas por muchos factores, como la distancia del cultivo a la zanja, modo de aplicación, condiciones climáticas, etc. Esto supone una preocupación si no se siguen ciertos protocolos de aplicación o se desconocen los efectos potenciales en los ecosistemas acuáticos (Stoorvogel y col., 2003; Pisa y col., 2015), tal es el caso del camarón de río, el cual con frecuencia se encuentra en ríos cercanos a los campos de arroz, donde el fipronil e imidacloprid son de amplio uso (Wasiw, 2017).

Se han publicado varios estudios de laboratorio sobre la toxicidad del imidacloprid en una variedad de invertebrados acuáticos y el organismo de prueba estándar, Daphnia magna Straus, 1820, quien es menos tóxico a los neonicotinoides (imidacloprid) en comparación a otros invertebrados (Beketov y Liess, 2008; Escobar-Chávez, Alvariño y Iannacone, 2019). Una $C_{50}$ aguda de alrededor de $7000 \mu \mathrm{g} \cdot \mathrm{L}^{-1}$ representa varios órdenes de magnitud por encima de las concentraciones efectivas encontradas para otros invertebrados. Esto implica que no siempre $D$. magna se puede utilizar como un organismo de prueba sensible y protector para toda la trama trófica acuática, a diferencia de C. caementarius que evidenció una mayor sensibilidad (Ngim y Crosby, 2001).

Aunque no se cuenta con información disponible de la toxicidad de estos insecticidas en el $C$. caementarius, existen estudios como el de Van Dijk, Van Staalduinen y Sluijs (2013), donde se evaluó la abundancia de macroinvertebrados acuáticos y las concentraciones de imidacloprid en aguas superficiales. Se observó que la abundancia de macroinvertebrados disminuye al aumentar la concentración del imidacloprid en el medio acuático (Beketov y col., 2013).

De acuerdo a las magnitudes del nivel de riesgo obtenidos de la literatura (i.e. $R Q \geq 1$ de alto riesgo, $0,1 \leq \mathrm{RQ}<1$ de riesgo medio, $0,01 \leq \mathrm{RQ}<0,1$ de bajo riesgo) (Sánchez-Bayo, Baskaran y Kennedy, 2002), los dos insecticidas presentaron un alto riesgo al ecosistema acuático en base a los efectos letales y subletales de $C$. caementarius.

En general, los resultados de la presente investigación y de la literatura publicada indican que ambos insecticidas tienen el potencial de causar daños significativos a los ecosistemas acuáticos al causar efectos negativos en individuos y poblaciones de invertebrados acuáticos en concentraciones muy bajas (Chaton y col., 2002). Para C. caementarius existiría un mayor riesgo en los periodos de diciembre a 
marzo y según la normatividad peruana, esta especie se encuentra en periodo de veda, debido a que durante esta etapa se observa una mayor presencia de huevos y hembras ovígeras (Baltazar y Colón, 2014).

\section{Conclusiones}

El bioindicador C. caementarius permite evaluar el riesgo ambiental del fipronil y el imidacloprid en el ecosistema acuático al emplear el cociente de riesgo a $96 \mathrm{~h}$ de exposición en base a la mortalidad en la respuesta letal, así como a la mortalidad y la subletalidad en base a la hipoactividad natatoria. El imidacloprid presentó un mayor riesgo para el ambiente acuático que el fipronil para la respuesta letal (mortalidad) y subletal (hipoactividad natatoria postlarval). La diferencia tóxica observada entre ambos insecticidas pudiera deberse a sus diferentes modos de acción. Este invertebrado nativo del Perú, C. caementarius, debería ser considerado como una especie sensible al momento de definir un ECA para la conservación del ambiente acuático, sobre todo en los periodos de enero a marzo, en las que según la normatividad peruana esta especie se encuentra en veda. Por ende, es recomendable continuar monitoreando la presencia de estos insecticidas en los cuerpos de agua dulceacuícolas costeras, y reducir el uso del fipronil y del imidacloprid en los cultivos agrícolas que los emplean.

\section{Referencias}

Al-Badran, A., M. Fujiwara y M. Mora (2019). «Effects of insecticides, fipronil and imidacloprid, on the growth, survival, and behavior of brown shrimp Farfantepenaeus aztecus». En: PloS one 14.10, e0223641. Online:https: / / bit.ly / $35 \mathrm{AhNqm}$.

Al-Badran, A. y col. (2018). «Lethal and sub-lethal effects of the insecticide fipronil on juvenile brown shrimp Farfantepenaeus aztecus». En: Scientific reports 8.1, 1-12. Online:https: / / go . nature.com/3nIvSbg.

Baltazar, G. y C. Colón (2014). «Algunos aspectos biológicos pesqueros de Cryphiops Caementariusçamarón de rio"(Molina, 1782) en la cuenca baja del río Mala». En: Científica 11.1, 30-45. Online:https://bit.ly/39kq3vm.
Beketov, M. A. y M. Liess (2008). «Potential of 11 pesticides to initiate downstream drift of stream macroinvertebrates». En: Archives of environmental contamination and toxicology 55.2, 247-253. Online:https:/ / bit.ly/3i92WrL.

Beketov, M. y col. (2013). «Pesticides reduce regional biodiversity of stream invertebrates». En: Proceedings of the National Academy of Sciences 110.27, 11039-11043. Online:https : / / bit . ly / 3bA3O7q.

Bonmatin, J. y col. (2015). «Worldwide integrated assessment of the impact of systemic pesticides on biodiversity and ecosystems.» En: Environmental Science and Pollution Research 22.1, 35-67. Online:https://bit.ly/3oJFp3a.

Campos, S. y col. (2017). «Monitoreo biológico y poblacional del recurso "camarón de río" Cryphiops caementarius (Molina, 1782) en los ríos MajesCamaná y Ocoña. 2015.» En: Informe Instituto del Mar Perú 44.3, 442-448. Online:https: / / bit.ly / 2LP3pDh.

Chaton, P. y col. (2002). «Toxicity and bioaccumulation of fipronil in the nontarget arthropodan fauna associated with subalpine mosquito breeding sites». En: Ecotoxicology and Environmental Safety 52.1, 8-12. Online:https://bit.ly/3nGn1Xs.

Cox, L. y col. (1998). «Sorption of imidacloprid on soil clay mineral and organic components». En: Soil Science Society of America Journal 62.4, 911-915. Online:https:/ / bit.ly/39vf5DB.

De la Torre, A., J. Ñuñoz y M. Carballo (2004). Curso sobre toxicología Ambiental y Seguridad Qímica. Evaluación Medioambiental y Ecotoxicológica. Inf. téc. Sanidad Ambiental, 20-24. Online:https: / / bit.ly/2V7W56Y.

EC (2011). Technical Guidance for Deriving Environmental Quality Standards. Common Implementation Strategy for the Water Framework Directive (2000/60/EC). Ed. por European Communities. 27. Brussels, Belgium: European Commission.

Escobar-Chávez, C., L. Alvariño y J. Iannacone (2019). «Evaluation of the Aquatic Environmental Risk of the mixture of the pesticides imidacloprid (insecticide) and propineb (Fungicide) in Daphnia magna Straus, 1820». En: Paideia XXI 9.2, 301-332. Online:https:/ / bit.ly /39rzMQL.

Gangwar, R. y col. (2016). «Effect of surfactant on the efficacy of insecticides against onion thrips (Thrips tabaci)». En: Indian Journal of Agricultural Sciences 86.6, 757-61. Online:https: / / bit.ly / $39 x V 8 f w$. 
Goff, A. y col. (2017). «The effects of fipronil and the photodegradation product fipronil desulfinyl on growth and gene expression in juvenile blue crabs, Callinectes sapidus, at different salinities». En: Aquatic Toxicology 186, 96-104. Online:https://bit.ly/2XCIiXM.

Hook, S. y col. (2018). "The impacts of modernuse pesticides on shrimp aquaculture: An assessment for north eastern Australia». En: Ecotoxicology and environmental safety 148, 770-780. Online:https://bit.ly/3i7not7.

INEI (2018). Anuario de Estadísticas Ambientales. Inf. téc. Lima. 717 pp.: Instituto Nacional de Estadística e Informática.

Key, P. y col. (2007). "Toxicity of three pesticides individually and in mixture to larval grass shrimp (Palaemonetes pugio)». En: Ecotoxicology and Environmental Safety 68.2, 272-277. Online:https:/ / bit.ly/3nFrMAO.

MINAGRI (2020). «Decreto Supremo que aprueba la modificación del Texto Único de Procedimientos Administrativo -TUPA del Servicio Nacional de Sanidad Agraria -SENASA». En: Decreto Supremo $N^{\circ}$ 001-2020-MINAGRI. Ed. por Ministerio de Agricultura y Riego. Disponible en https: / / bit.ly/3aNurTt.

Mendoza-Rodríguez, R. (2009). «Toxicidad aguda del sulfato de cobre en postlarvas de camarón cryphiops caementarius». En: Archivos de zootecnia 58.221, 103-110. Online:https: / / bit.ly / 2LPEJec.

Ngim, K. y D. Crosby (2001). "Abiotic processes influencing fipronil and desthiofipronil dissipation in California, USA, rice fields». En: Environmental Toxicology and Chemistry: An International Journal 20.5, 972-977. Online:https : / / bit . ly / 3nM77Lb.

Omar, H. y col. (2016). "Acute water column effects concentrations (LC50, LC90) for three commonly used insecticides, two Neonicotinoids (Acetamiprid and Imidacloprid), and a recently registered Phenylpyrazole (Fipronil), exposed to common commercially cultured shrimp (Marsupenaeus japonicus)». En: 55th Annual Meeting of the Society of Toxicology. Online:https: / / bit.ly / 3e9zAr0.

Pathak, M. y col. (2018). «Evaluation of Different Insecticides against Onion Thrips in Onion Seed Production». En: International Journal of Current Microbiology and Applied Sciences 7.7, 4204-4207. Online:https://bit.ly/2LvxFU6.
Pisa, L. y col. (2015). «Effects of neonicotinoids and fipronil on non-target invertebrates». En: Environmental Science and Pollution Research 22.1, 68-102. Online:https://bit.ly/35EpThw.

Reyes, W (2011). «Crecimiento, reproducción y supervivencia de hembras del camarón de río Cryphiops caementarius criados en recipientes individuales». En: Sciéndo 14.1, 77-88. Online:https://bit.ly/2X9y4Pw.

Reyes, Walter (2018). "El síndrome de la ecdisis incompleta en machos adultos de Cryphiops caementarius (Crustacea: Palaemonidae) y sus consecuencias en cultivo intensivo». En: Revista de Investigaciones Veterinarias del Perú 29.1, 368-374. Online:https:/ / bit.ly/3qcOGky.

Rice, E., R. Baird y A. Eaton (2017). Standard Methods for the examination of water and wastewater, 23rd Ed. Denver, USA: American Public Health Association, American Water Works Association, Water Environment Federation.

Romero-Camarena, H., M. Zelada y V. Álvarez (2013). «Producción larval del Camarón de río (Cryphiops caementarius) en condiciones de laboratorio, Huacho, Perú». En: Infinitum 3.1, 35-40. Online:https://bit.ly/3nBzR9C.

Sánchez-Bayo, F., S. Baskaran e I. Kennedy (2002). «Ecological relative risk (EcoRR): another approach for risk assessment of pesticides in agriculture». En: Agriculture, Ecosystems y Environment 91.1-3, 37-57. Online:https : / / bit . ly / 2XFfIEY.

Shan, Z. y col. (2003). «Impact of fipronil on crustacean aquatic organisms in a paddy fieldfishpond ecosystem». En: Bulletin of environmental contamination and toxicology 70.4, 746-752. Online:https://bit.ly/2XKhkx7.

Sluijs, J. Van der y col. (2015). "Conclusions of the Worldwide Integrated Assessment on the risks of neonicotinoids and fipronil to biodiversity and ecosystem functioning». En: Environ Sci Pollut Res 22, 148-154. Online:https: / / bit . ly / 3qmv4ui.

Smit, C. y col. (2015). «Ecotoxicity of imidacloprid to aquatic organisms: derivation of water quality standards for peak and long-term exposure». En: Human and Ecological Risk Assessment: An International Journal 21.6, 1608-1630. Online:https: / / bit.ly/3oFlOBb.

Sohn, L. y col. (2018). «Exposure to a nicotinoid pesticide reduces defensive behaviors in a nontarget organism, the rusty crayfish Orconectes 
rusticus». En: Ecotoxicology 27.7, 900-907. Online:https://bit.ly/2XFqNpF.

Sotelo-Vásquez, D. y J. Iannacone (2019). «Acute toxicity of three pesticides (Butachlor, copper oxychloride and chlorpyrifos) on the marine benthic amphipod Apohyale grandicornis (Kroyer, 1945) (Crustacea: Hyalidae)». En: Biotempo (Lima) 16, 241-256. Online:https:/ / bit.ly / 2MUROmL.

Stevens, M. y col. (2011). «The acute toxicity of fipronil to two non-target invertebrates associated with mosquito breeding sites in Australia». En: Acta tropica 117.2, 125-130. Online:https: / / bit. ly/3qlZWv7.

Stoorvogel, J. y col. (2003). Plaguicidas en el medio ambiente. Los Plaguicidas. Impactos en produccion, salud y medio ambiente en Carchi, Ecuador. Lima: Centro International de la Papa.

Tennekes, H. (2018). «Fipronil in surface water: an environmental calamity remaining under radar in the Netherlands». En: Journal of Ecology and Toxicology 2.1. Online:https://bit.ly/2XkRLnH.

Van Dijk, T., M. Van Staalduinen y J. Van der Sluijs (2013). "Macro-invertebrate decline in surface water polluted with imidacloprid». En: PloS one 8.5, e62374. Online:https://bit.ly/3oPaqmk.

Vijver, M. y P. Van den Brink (2014). «Macroinvertebrate decline in surface water polluted with imidacloprid: a rebuttal and some new analyses». En: PLoS One 9.2, e89837. Online:https:/ / bit.ly/2N1Q4rX.

Wasiw, J. y V. Yépez (2015). «Evaluación poblacional del camarón Cryphiops caementarius en ríos de la costa sur del Perú». En: Revista de investigaciones veterinarias del Perú 26.2, 166-181. Online:https://bit.ly/39wCvZb.

Wasiw J.and Yépez, V. (2017). «Evolución de la condición poblacional del camarón Cryphiops caementarius en el Río Cañete (2000-2015)». En: Revista de Investigaciones Veterinarias del Perú 28.1, 13-32. Online:https://bit.ly/2N5dg8S. 